\title{
Milk-derived hexapeptide PGPIPN prevents and attenuates acute alcoholic liver injury in mice by reducing endoplasmic reticulum stress
}

\author{
QIA XU $^{1 *}$, HAO XI ${ }^{1,2^{*}}, \mathrm{XI} \mathrm{CHEN}^{1 *}, \mathrm{YIN} \mathrm{XU}^{3}, \mathrm{PENG} \mathrm{WANG}^{1}, \mathrm{JINGWEN} \mathrm{LI}^{1}$, \\ WENMEI WEI ${ }^{1}$, FANG GU ${ }^{1}$ and YIDE QIN ${ }^{1}$ \\ ${ }^{1}$ School of Basic Medical Sciences, Anhui Medical University, Hefei, Anhui 230032; \\ ${ }^{2}$ Department of Pathology, The Second People's Hospital of Hefei, Hefei, Anhui 230011, P.R. China; \\ ${ }^{3}$ Huffington Center on Aging, Baylor College of Medicine, Houston, TX 77030, USA
}

Received January 20, 2020; Accepted May 27, 2020

DOI: $10.3892 / \mathrm{ijmm} .2020 .4643$

\begin{abstract}
Bioactive peptides are an emerging area of biomedical research in the study of numerous human diseases, including acute alcoholic liver injury (AALI). To study the role and mechanism of the milk-derived hexapeptide Pro-Gly-Pro-Ile-Pro-Asn (PGPIPN) in preventing and reducing AALI, the present study established a mouse model of AALI. PGPIPN was used as a therapeutic drug, and glutathione (GSH) was used as a positive control. The body and liver weights of mice were measured, and the liver indexes were calculated to observe mice health. The pathological morphology of liver tissues stained with hematoxylin and eosin were examined to analyze hepatic injury, and hepatocyte apoptosis was measured with a TUNEL assay. The concentrations or activities of alanine aminotransferase (ALT), aspartate aminotransferase, tumor necrosis factor- $\alpha$, interleukin (IL)-1 $\beta$, IL-6, triglyceride, total cholesterol, malondialdehyde, superoxide dismutase and GSH peroxidase (GSH-PX) were detected in serum and/or liver homogenates. The $78 \mathrm{kDa}$ glucose-regulated protein (GRP78), protein kinase R-like (PKR) endoplasmic reticulum kinase (PERK), phosphorylated (p)-PERK, eukaryotic initiation factor $2 \alpha$ (eIF-2 $\alpha$ ), p-eIF- $2 \alpha$, inositol-requiring enzyme $1 \alpha$ (IRE-1 $\alpha$ ), spliced X-box binding protein 1 (XBP-1s), C/EBP homologous protein (CHOP), caspase- 3 and cleaved caspase- 3 proteins associated with endoplasmic reticulum stress in hepatocytes were assessed
\end{abstract}

Correspondence to: Professor Yide Qin or Dr Fang Gu, School of Basic Medical Sciences, Anhui Medical University, 81 Meishan Street, Hefei, Anhui 230032, P.R. China

E-mail: yideqin@ahmu.edu.cn

E-mail:wsxgf2013@163.com

${ }^{*}$ Contributed equally

Key words: PGPIPN, acute alcoholic liver injury, apoptosis, cytokine, endoplasmic reticulum stress by western blotting, and RNA levels of XBP-1s, CHOP and caspase-3 genes were assessed by reverse transcription-quantitative PCR. The results suggested that PGPIPN attenuated alcoholic hepatocyte damage in animal models and reduced hepatocyte oxidative stress in a dose-dependent manner. Moreover, PGPIPN reduced endoplasmic reticulum stress by regulating the expression levels of p-PERK, p-eIF- $2 \alpha$, XBP-1s, CHOP, caspase- 3 and cleaved caspase- 3 . Collectively, the present results indicated that PGPIPN, as a potential therapeutic drug for AALI, exerted a protective effect on the liver and could reduce liver damage.

\section{Introduction}

Alcoholic liver disease is associated with high morbidity rates worldwide. Alcohol consumption accounts for $3.8 \%$ of annual global mortality worldwide, and the majority of these deaths are due to alcoholic liver disease (1). Furthermore, alcoholic liver injury (ALI) is a major cause of morbidity and mortality in industrialized and developing countries, especially China (2). ALI causes a series of changes, progressing from steatosis to hepatitis, fibrosis, cirrhosis and ultimately hepatocellular carcinoma (3). Heavy alcohol consumption ( $>60 \mathrm{~g}$ /day) causes acute ALI (AALI) in the short term (4). Moreover, AALI cannot be diagnosed clinically, thus patients are diagnosed in advanced stages of the disease (5). Currently, there are few treatments, and the most common steroid treatment, corticosteroids, is not satisfactory (6). In addition, cytokine therapy is expensive and difficult to perform $(7,8)$. Therefore, there is an urgent need to develop novel safe and effective drugs.

There are several unknown bioactive peptides in the human daily diet, such as milk and corn, which researchers have extracted from food and have shown to be beneficial to humans (9). Bioactive peptides derived from proteins are beneficial to human health, and were reported to possess numerous biological activities, such as anti-oxidation, anti-hypertension, anti-diabetes and immune regulation, and also play important roles in regulating immune responses and certain physiological functions in vivo (10-12). Previous studies revealed that bioactive peptides play an important role 
in early ALI and chronic alcoholic injury in mice $(13,14)$, and can promote alcohol clearance and bile acid metabolism, as well as reduce serum the levels or activities of total cholesterol (TC), triglyceride (TG), alanine aminotransferase (ALT) and aspartate aminotransferase (AST) (14).

PGPIPN (Pro-Gly-Pro-Ile-Pro-Asn) originates from $\beta$-casein (residues 63-68) in bovine milk (15). Generally, short peptides ( $\leq 7$ amino acids) can be absorbed directly via the digestive tract into the blood (16). PGPIPN-containing three prolines can resist hydrolysis by digestive enzymes in the gastrointestinal tract (17). PGPIPN has been reported to have immunoregulation and anticancer effects (15,18-20). For example, PGPIPN inhibited cell proliferation and induced cell apoptosis in the human ovarian cancer cell line SKOV 3 in vitro and reduced tumor growth rates in mice (21). Recent studies showed that PGPIPN can alleviate alcoholic fatty liver disease (13). Therefore, the aim of the present study was to investigate whether PGPIPN can alleviate AALI in mice. The results suggested that PGPIPN may be used as a potential treatment for AALI.

\section{Materials and methods}

Reagents. PGPIPN (purity $>99.5 \%$, confirmed by reversed phase-high-performance liquid chromatography) was supplied by Sangon Biotech Co., Ltd. Hematoxylin solution was purchased from Sigma-Aldrich (Merck KGaA). The $78 \mathrm{kDa}$ glucose-regulated protein (GRP78; cat. no. ab108615), C/EBP homologous protein (CHOP; cat. no. ab11419), caspase-3 (cat.no.ab4051), cleaved caspase-3 (cat.no.ab49822) and $\beta$-actin (cat. no. ab8227) antibodies were purchased from Abcam. Protein kinase R-like (PKR) endoplasmic reticulum kinase (PERK; cat. no. 3192), phosphorylated (p)-PERK (Thr980; cat. no. 3179), p-eukaryotic initiation factor $2 \alpha$ (p-eIF- $2 \alpha$; Ser51; cat. no. 3398), eIF-2 $\alpha$ (cat. no. 5324), inositol-requiring enzyme $1 \alpha$ (IRE-1 $\alpha$; cat. no. 3294) and spliced X-box binding protein 1 (XBP-1s; cat. no. 82914) antibodies were purchased from Cell Signaling Technology, Inc. Secondary antibodies [horseradish peroxidase (HRP)-conjugated goat anti-mouse immunoglobulin $\mathrm{G}$ ( $\mathrm{IgG})$, cat. no. GAM-HRP; HRP-conjugated goat anti-rabbit IgG, cat. no. GAR-HRP] and Super Signal West Pico kit (ECL Chromogenic kit) was purchased from Thermo Fisher Scientific, Inc.

Alcohol-induced animal models and pharmacological intervention. A total of 60 healthy male Kunming mice (weight, 18-22 g; 6-8 weeks old) were purchased from Anhui Medical Experimental Animal Center (batch no. 0000469). All animal experiments were performed under procedures approved by the Institutional Animal Care and Use Committee of Anhui Medical University (approval no. LLSC20180132). All methods and protocols used in the relevant studies, including animal and related studies in vivo, were conducted in accordance with the relevant guidelines and regulations of the Committee's protocol. All mice were housed in a specific pathogen-free sterile rooms at $22 \pm 2^{\circ} \mathrm{C}, 40-60 \%$ relative humidity and a $12-\mathrm{h}$ light/dark cycle in the Anhui Medical Laboratory Animal Center. Animal suffering in experiments was alleviated as much as possible. All mice, with free access to food and drinking water, were acclimatized for 1 week, then divided into six groups (n=10/group): Control, model, glutathione (GSH), low-dose PGPIPN (PGPIPN L), moderate-dose PGPIPN (PGPIPN M) and high-dose PGPIPN (PGPIPN H). The test period was 10 days. PGPIPN L, PGPIPN M and PGPIPN H groups were treated with PGPIPN at $0.04,0.4$ and $4 \mathrm{mg} / \mathrm{kg}$ body weight via oral gavage each day, respectively. The control and model groups were treated with the same volume of saline $(250 \mu \mathrm{l})$ instead of PGPIPN. The GSH group was treated with GSH instead of PGPIPN as a positive control at $20 \mathrm{mg} / \mathrm{kg}$ body weight. During the last 3 days, the model, PGPIPN L, PGPIPN M, PGPIPN $\mathrm{H}$ and GSH groups were administered with $9.5 \mathrm{~mol} / \mathrm{l}$ ethanol at $15 \mathrm{ml} / \mathrm{kg}$ body weight for oral gavage $2 \mathrm{~h}$ after the aforementioned peptide infusion, and the control group was administered the same volume of saline. At the end of the experiment, all mice were anesthetized, and serum and liver samples were weighed, collected and stored. The liver index was calculated, liver index $(\%)=$ liver weight/body weight. Some liver tissues were fixed in paraffin after $10 \%$ neutral buffered formalin, and the remaining tissues were cryopreserved in liquid nitrogen for subsequent experiments.

Morphological observation and calculation of liver injury and hepatocyte apoptosis. Mice liver tissues were fixed with $4 \%$ paraformaldehyde solution at room temperature for $24 \mathrm{~h}$, dehydrated with alcohol, embedded in paraffin and prepared into tissue sections. Liver tissues stained with hematoxylin and eosin (H\&E) were observed and analyzed under an optical microscope at x200 magnification. Hepatocyte apoptosis of liver tissues was analyzed with a TUNEL assay according to the manufacturer's instructions (cat. no. 12156792910; Roche Diagnostics $\mathrm{GmbH}$ ). Mice liver tissues were fixed with $4 \%$ paraformaldehyde solution at room temperature for 20 min. Subsequently, paraffin sections were made, followed by dewaxing, hydration and penetration, then stained with TUNEL reagent $\left(\mathrm{TdT}+\right.$ fluorescein-labeled dUTP) at $37^{\circ} \mathrm{C}$ for $1 \mathrm{~h}$ in the dark and humidified atmosphere. The nuclei were stained with methyl green at room temperature for a few seconds. The number of fields of view observed by fluorescent microscopy was 200-500 cells. Apoptosis was quantified using ImageJ software (version 1.44; National Institutes of Health). The apoptosis index (AI) was calculated as following formula: $\mathrm{AI}=$ (number apoptotic cells/total number of cells) $\mathrm{x} 100 \%$.

Analysis of biochemical materials, cytokines and enzyme activities related to lipid metabolism, oxidation and liver injury in serum and/or liver tissues of animal models. Serum TG, TC, ALT and AST concentrations or activities were determined using a Roche Cobas automated biochemical analyzer (Roche Diagnostics). A $10 \% \mathrm{w} / \mathrm{v}$ liver homogenate was prepared from fresh liver tissue. The TG. (cat. no. F001-1-1), malondialdehyde (MDA; cat. no. A003-1-1), superoxide dismutase (SOD; cat. no. A001-1-1) and glutathione peroxidase (GSH-PX; cat. no. A005-1-2) concentrations or activities in liver were quantified using the aforementioned commercially available kits (Nanjing Jiancheng Bioengineering Institute) according to the manufacturer's instructions. Tumor necrosis factor- $\alpha$ (TNF- $\alpha$; cat. no. XFFM1870), interleukin (IL)-1 $\beta$ (cat. no. RIA-127) and IL-6 (cat. no. XEFM028D) were quantified by radioimmunoassay using commercially available kits (Shanghai Xinfan Biological Technology Co., Ltd.) according to the manufacturer's instructions. 
Reverse transcription-quantitative PCR (RT-qPCR). At the end of the experiment, all mice liver tissues were harvested, in which the total RNAs were extracted with TRIzol ${ }^{\circledR}$ reagent and RNA extraction buffer according to the manufacturer's instructions (cat. no. 15596026; Invitrogen; Thermo Fisher Scientific, Inc.). RNA purity and concentration were determined by ultraviolet spectrophotometry. According to the RNA template and primer [Oligo(dT)], the first strand cDNA was synthesized in a reverse transcription reaction including buffers, dNTPs, AMV reverse transcriptase, recombinant RNasin and total RNA, according to the manufacturer's instructions (Revert Aid First Strand cDNA Synthesis kit; cat. no. K1621; Thermo Fisher Scientific, Inc.). The reverse transcription reaction conditions were $42^{\circ} \mathrm{C}$ for $1 \mathrm{~h}$ and $70^{\circ} \mathrm{C}$ for $5 \mathrm{~min}$. Hepatic mRNA expression levels of caspase-3, XBP-1s and CHOP were measured by RT-qPCR using the Applied Biosystems 7500 Real-Time PCR system (Applied Biosystems; Thermo Fisher Scientific, Inc.). The following primer pairs (synthesized by Sangon Biotech Co., Ltd.) were used for the qPCR: Caspase-3 forward, 5'-ATG GAGAACAACAAAACCTCAGT-3' and reverse, 5'-TTGCTC CCATGTATGGTCTTTAC-3'; XBP-1s forward, 5'-TGCTGA GTCCGCAGCAGGTG-3' and reverse, 5'-GCTGGCAGG CTCTGGGGAAG-3'; CHOP forward, 5'-CTGGAAGCCTGG TATGAGGAT-3' and reverse, 5'-CAGGGTCAAGAGTAG TGAAGGT-3' and $\beta$-actin forward, 5'-GAAATCGTGCGT GACATCAAAG-3' and reverse, 5'-TGTAGTTTCATGGAT GCCACAG-3'. $\beta$-actin was used as the housekeeping gene.

RT-qPCR was performed using TB Green Premix Ex Taq (cat. no. RR420L; Takara Bio Inc.) according to the manufacturer's instructions. The following thermocycling conditions were used for the qPCR: $95^{\circ} \mathrm{C}$ for $30 \mathrm{sec}$ and 40 cycles of $95^{\circ} \mathrm{C}$ for $5 \mathrm{sec}$ and $60^{\circ} \mathrm{C}$ for $34 \mathrm{sec}$. At the end of PCR cycling steps, data for each sample were displayed as a melting curve. The specificity of the amplified products was confirmed using melting curve analysis. For each target gene, mRNA expression levels were calculated using the $2^{-\Delta \Delta \mathrm{Cq}}$ method ( $\Delta \mathrm{Cq}=$ target gene $\mathrm{Cq}-\beta$-actin $\mathrm{Cq}$ value) (22). All reactions were performed in triplicate, and a mixture lacking a complementary DNA template was used as the negative control. In total, two independent experiments were run.

Western blotting. The liver tissues of the mice were lysed using RIPA buffer (cat. no. R0010; Beijing Solarbio Science \& Technology Co., Ltd.) with $1 \%$ phenylmethanesulfonyl fluoride. Protein concentration was measured using a bicinchoninic acid protein assay kit (cat. no. P0012; Beyotime Institute of Biotechnology). Proteins isolated from liver tissues were separated by SDS-PAGE [5\% stacking gel, 10 or $12 \%$ lower gel $(\mathrm{w} / \mathrm{v}) ; 22.5 \mu \mathrm{g}$ protein in $15 \mu 1$ loaded per lane], and subsequently transferred to PVDF membranes. Following blocking with 5\% (w/v) dry skim milk for $2 \mathrm{~h}$, then immunoblots were performed using a standard protocol (23). The membranes were incubated with primary antibodies and later incubated with HRP-conjugated secondary antibodies. The operating procedures were undertaken in biochemical incubator at room temperature $\left(22^{\circ} \mathrm{C}\right)$. The following primary antibodies were used: Rabbit or mouse monoclonal antibodies [GRP78, PERK, p-PERK (Thr980), eIF-2 $\alpha$, p-eIF-2 $\alpha$ (Ser51), IRE-1 $\alpha$, XBP-1s and CHOP; 1:1,000] and rabbit polyclonal antibodies (caspase-3 and cleaved caspase-3; 1:500; $\beta$-actin;
1:1,000). Secondary antibodies used were goat anti-mouse and goat anti-rabbit IgG, diluted at 1:10,000. Proteins were detected using the ECL system and exposed in a chemiluminescent imaging system (Clinx Science Instruments Co., Ltd.), and obtained images were quantitatively analyzed using ImageJ software (version1.48u; National Institutes of Health). $\beta$-actin was used as a control. All reactions were performed in triplicate, and two independent experiments were run.

Statistical analysis. Data are presented as the mean \pm SD. Statistical analyses were performed with SPSS version 20.0 (IBM Corp.) using one-way ANOVA followed by Tukey's post hoc test. $\mathrm{P}<0.05$ was considered to indicate a statistically significant different.

\section{Results}

PGPIPN prevents and reduces AALI in model animals. To investigate the specific role of PGPIPN in the treatment of AALI, a mice model of AALI was successfully established. The body weights of mice in the model group were significantly lower compared with the control group, but the hepatic weights and liver indexes were significantly higher compared with the control group (Fig. 1A-C). Therefore, AALI resulted in body weight loss and increased hepatic weight and liver index. However, PGPIPN relieved AALI symptoms and antagonized the effects of alcohol in a dose-dependent manner, which was similar to that of GSH as a positive control (Fig. 1A-C). Compared with the control group, the activities of ALT and AST in the mice serums of the model group were significantly higher (Fig. 1D). Compared with the model group, PGPIPN significantly decreased the activities of ALT and AST in a dose-dependent manner. This suggested that PGPIPN effectively reduced liver inflammation.

The effect of PGPIPN on mouse liver was analyzed via histological observation of liver tissues stained with H\&E under light microscopy. It was found that the cells of control group were arranged in a radial line, the structures of hepatic lobule were intact, the hepatocyte nucleus and cell boundaries were clear, and no necrosis was observed (Fig. 2A). In the model group, the hepatic cord arrangements were disordered and there were inflammatory cell infiltrations in the hepatocyte spaces (Fig. 2B). Furthermore, there were several swollen hepatocytes and vacuolization of the cytoplasm, as well as partial atrophied hepatocyte nuclei, in which these nuclei even disappeared and partial necrosis (Fig. 2B). In low- and medium-dose PGPIPN groups, hepatocyte swellings were alleviated, inflammatory cell infiltrations were reduced, and hepatocyte nuclear atrophy, disappearance and vacuolization were observed (Fig. 2D and E). In the high-dose PGPIPN group, the hepatic cord arrangements and hepatocyte structures were normal, and no obvious inflammatory cell infiltrations and cytoplasmic vacuolization were observed (Fig. 2F), which was similar to the GSH group (Fig. 2C).

A TUNEL assay was performed on the liver tissues of mice. TUNEL-positive cells in the liver tissues of the model group (Fig. 3Ab) were markedly increased compared with the control group (Fig. 3Aa). Compared with the model group, PGPIPN decreased TUNEL-positive cells in the liver tissues (Fig. 3Ad-f), of which moderate- and high-dose 

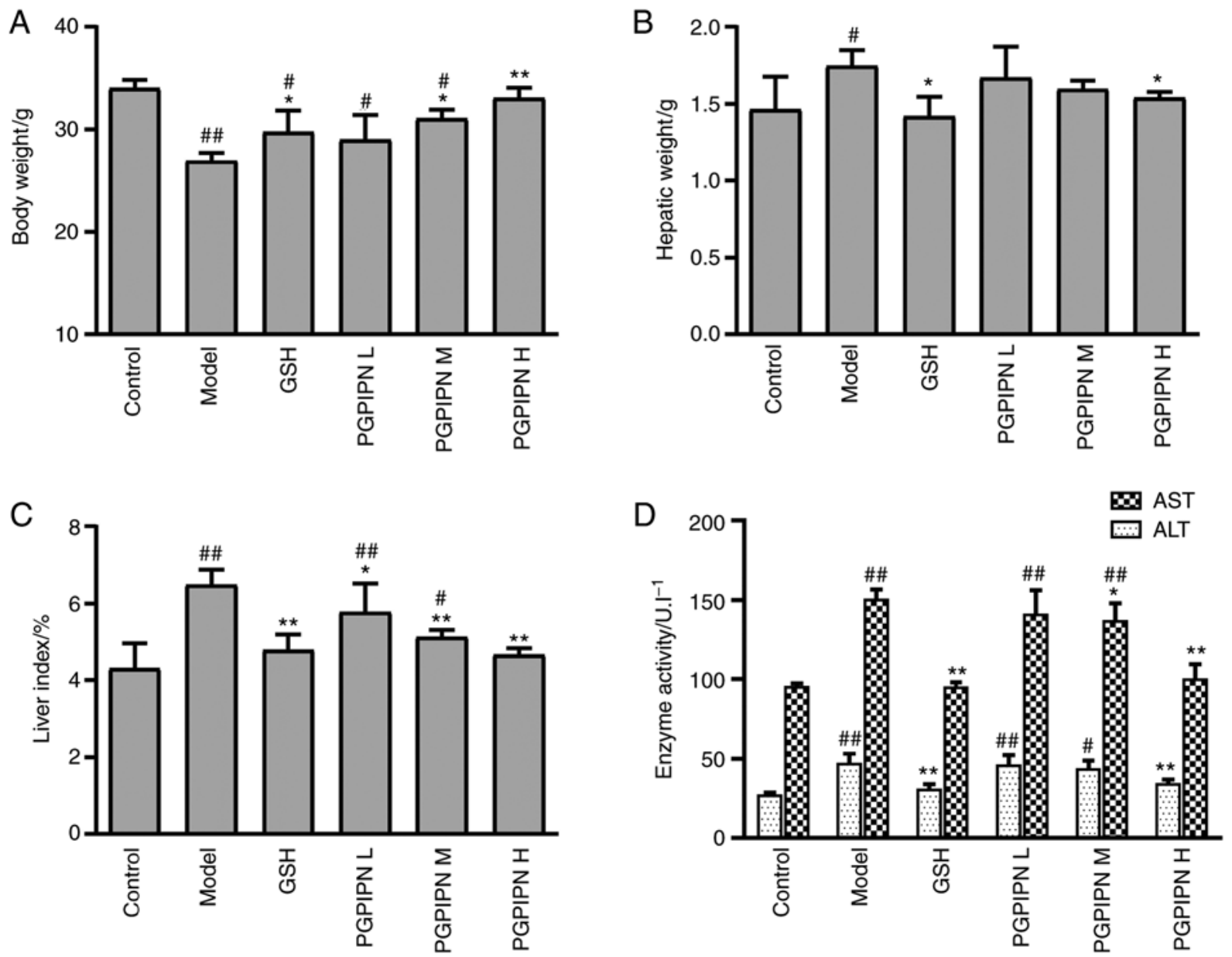

Figure 1. PGPIPN attenuates the liver index and serum ALT and AST activity in mice. (A) Body weight. (B) Liver weight. (C) Liver index. (D) Serum ALT and AST activity. Data are presented as mean \pm standard deviation of ten mice in each group. ${ }^{~} \mathrm{P}<0.05$ and ${ }^{* *} \mathrm{P}<0.01$ vs. model. ${ }^{\#} \mathrm{P}<0.05$ and ${ }^{\# \#} \mathrm{P}<0.01$ vs. control. ALT, alanine aminotransferase; AST, aspartate aminotransferase; PGPIPN, Pro-Gly-Pro-Ile-Pro-Asn; L, low dose; M, medium dose; H, high dose; GSH, glutathione.

A

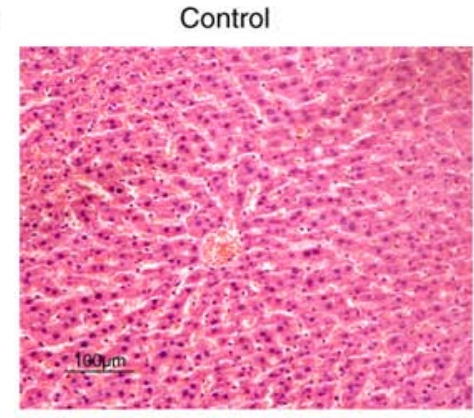

D

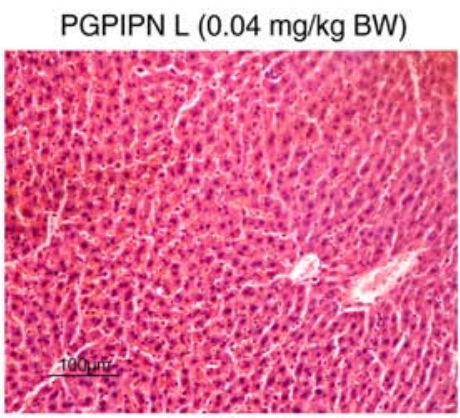

B

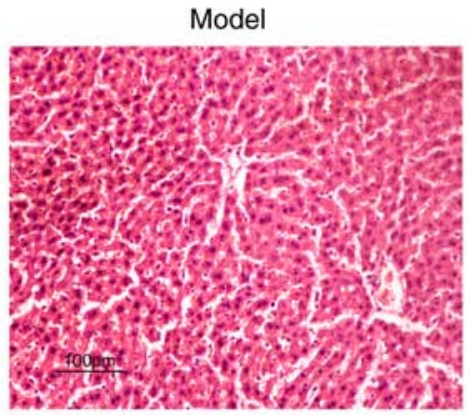

E

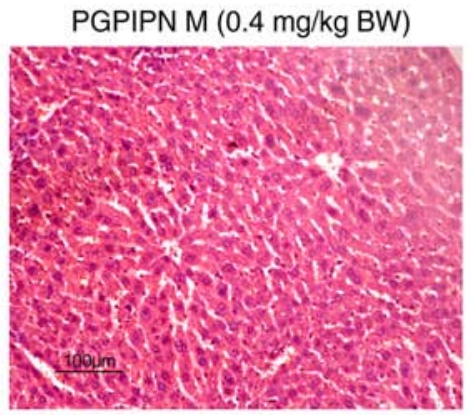

C

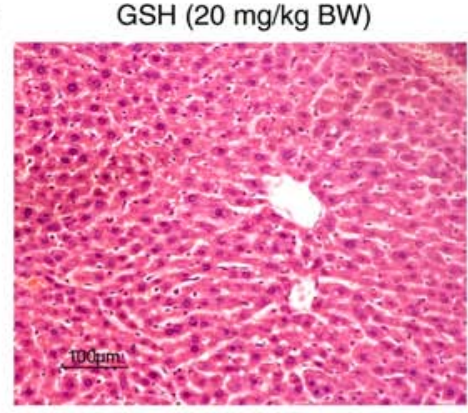

$\mathrm{F}$

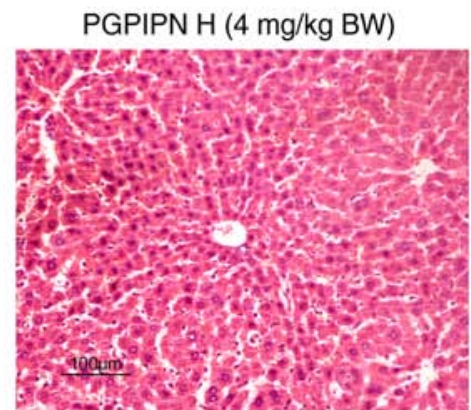

Figure 2. PGPIPN attenuates the pathological changes of mice liver tissues in acute alcoholic liver injury. Hematoxylin and eosin staining in (A) Control, (B) model, (C) GSH, (D) PGPIPN L, (E) PGPIPN M and (F) PGPIPN H groups. Magnification, x200. PGPIPN, Pro-Gly-Pro-Ile-Pro-Asn; L, low dose; $\mathrm{M}$, medium dose; $\mathrm{H}$, high dose; GSH, glutathione; BW, body weight. 

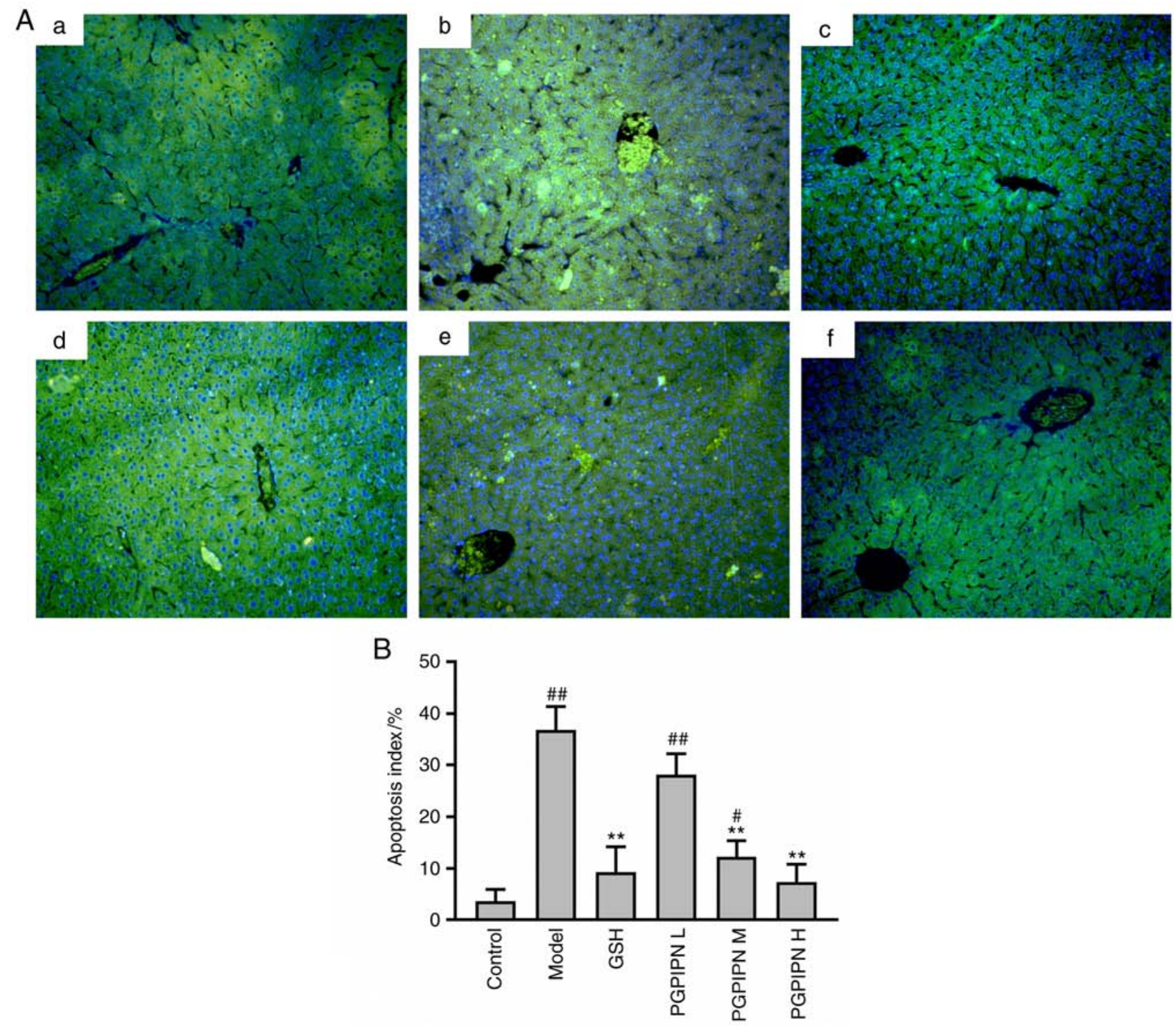

Figure 3. PGPIPN attenuates hepatocyte apoptosis of mice induced with alcohol-intake. (A) Liver tissues stained with a TUNEL kit (fluorescein-dUTP) and observed under an inverted fluorescence microscope. a, Control group; b, Model group; c, GSH group; d, PGPIPN L group; e, PGPIPN M group; f, PGPIPN H group. Magnification, $\mathrm{x} 200$. (B) Quantification of apoptosis using ImageJ software. Data are presented as the mean \pm standard deviation of ten mice in each group. ${ }^{* *} \mathrm{P}<0.01$ vs. model. ${ }^{\#} \mathrm{P}<0.05$ and ${ }^{\# \#} \mathrm{P}<0.01$ vs. control. PGPIPN, Pro-Gly-Pro-Ile-Pro-Asn; L, low dose; M, medium dose; H, high dose; GSH, glutathione; BW, body weight.

PGPIPN groups reached significant levels, and the decrease in high-dose PGPIPN group was greater compared with the GSH group (positive control; Fig. 3Ac). The AI was also significantly increased in the model group compared with control group (Fig. 3B). Compared with the model group, PGPIPN decreased hepatocyte apoptosis in a dose-dependent manner, and the effect was the most obvious in the PGPIPN H group (Fig. 3B).

PGPIPN decreases the levels of mouse pro-inflammatory cytokines. The levels of TNF- $\alpha$, IL- $1 \beta$ and IL- 6 were determined in mouse liver homogenates by radioimmunoassay. The results indicated that the TNF- $\alpha$, IL-1 $\beta$ and IL- 6 levels of model group mice were significantly increased compared with the control group. In addition, compared with the model group, PGPIPN significantly reduced the levels TNF- $\alpha$, IL-1 $\beta$ and IL-6 contents, similar to levels found the GSH group (Fig. 4).
PGPIPN attenuates alcohol-induced lipid metabolism and oxidative stress in mouse liver. The TG and TC in serum and liver homogenates of model group mice were significantly higher compared with the control group (Fig. 5A). Compared with the model group, PGPIPN significantly reduced the TG and TC levels in mice serum and liver, reductions of which were higher compared with the GSH group (positive control; Fig. 5A). Even at a low dose, PGPIPN had a significant effect on reducing aforementioned TG and TC levels. Thus, it was speculated that PGPIPN may attenuate liver damage by regulating lipid metabolism. The results also indicated that MDA levels in the liver tissues of the model group was significantly increased, and the activities of GSH-PX and SOD were significantly decreased compared with the control group (Fig. 5B-D). Furthermore, PGPIPN in medium and high doses significantly reduced MDA levels and increased of GSH-Px and SOD activity in mice liver tissues, effects of which were 

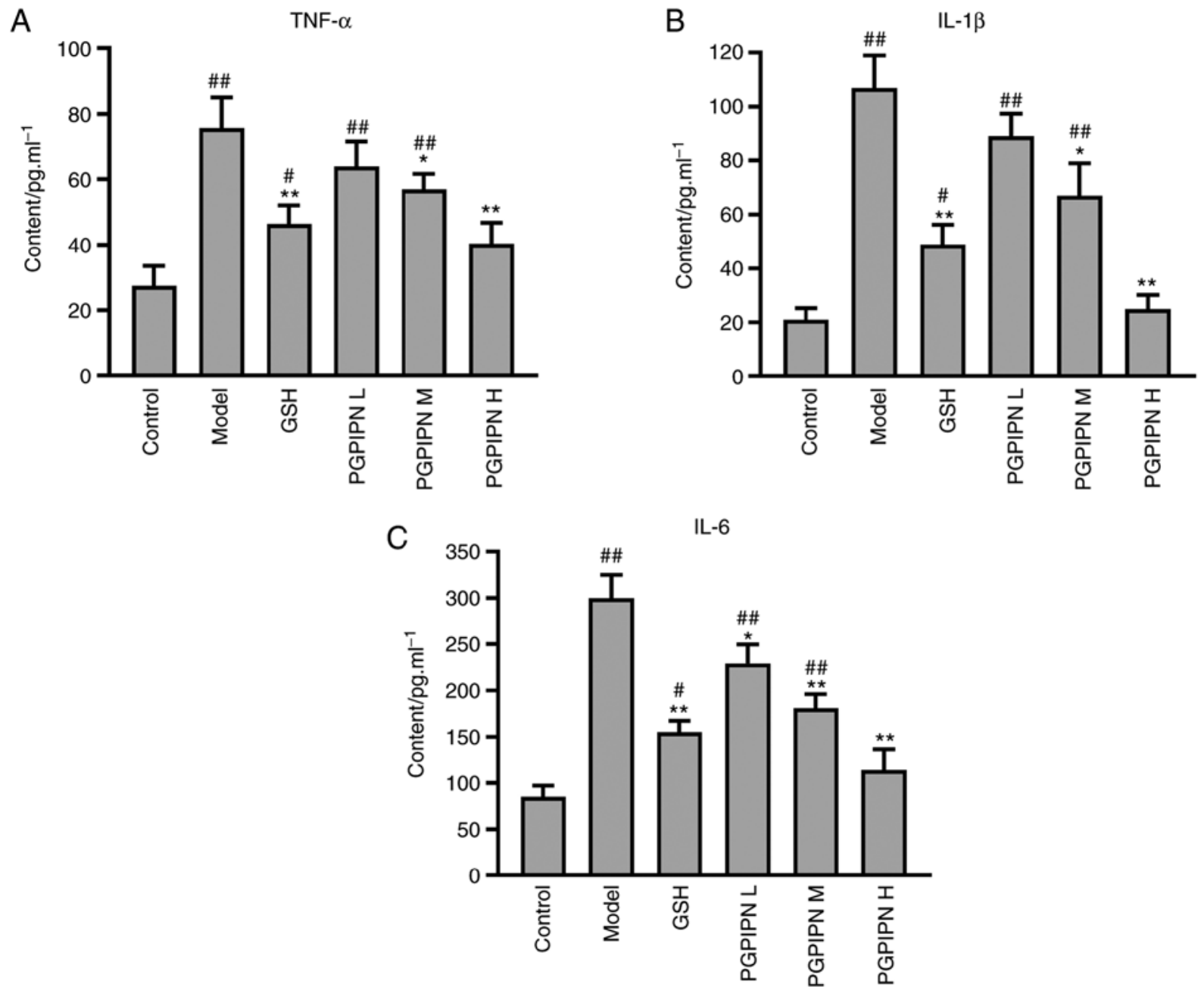

Figure 4. PGPIPN decreases the levels of inflammatory-related cytokines in mice liver tissues. (A) TNF- $\alpha$. (B) IL-1 $\beta$. (C) IL-6. Data are presented the mean \pm standard deviation of ten mice in each group. ${ }^{*} \mathrm{P}<0.05$ and ${ }^{* *} \mathrm{P}<0.01$ vs. model. ${ }^{\#} \mathrm{P}<0.05$ and ${ }^{\# \#} \mathrm{P}<0.01$ vs. control. TNF, tumor necrosis factor; IL, interleukin; PGPIPN, Pro-Gly-Pro-Ile-Pro-Asn; L, low dose; M, medium dose; H, high dose; GSH, glutathione.

similar to that of GSH group as a positive control. However, PGPIPN in low doses had no significant effect on these three indicators.

PGPIPN regulates the expression of genes associated with endoplasmic reticulum stress (ERS) in hepatocytes. The present study examined the mRNA expression levels of XBP-1s, CHOP and caspase-3 by RT-qPCR, which are three key indicators of ERS (24). Compared with control group mice, the mRNA expression levels of XBP-1s and CHOP were significantly increased in alcohol-induced model mice (Fig. 6). PGPIPN significantly decreased the expression levels of XBP-1s and CHOP compared with the model group (Fig. 6). The effects of PGPIPN on mRNA expression levels of XBP-1s and CHOP genes were dose-dependent. However, the mRNA expression of caspase-3 did not change significantly between the six groups, although the control group showed markedly lower expression (Fig. 6).

Western blotting was used to analyze GRP78, PERK, p-PERK, eIF-2 $\alpha$, p-eIF-2 $\alpha$, IRE-1 $\alpha$, XBP-1s, CHOP, caspase-3 and cleaved caspase- 3 proteins, which are related to ERS in liver tissues of mice (24). The protein expression levels of p-PERK, p-eIF-2 $\alpha$, XBP-1s, CHOP, caspase-3 and cleaved caspase-3 in the model group were significantly higher compared with the control group, but the expression levels of GRP78, PERK, eIF-2 $\alpha$ and IRE-1 $\alpha$ did show any significant changes (Fig. 7A, B and D). PGPIPN reduced the expression levels of p-PERK, p-eIF-2 $\alpha$, XBP-1s, CHOP, caspase-3 and cleaved caspase- 3 proteins compared with the model group in a dose-dependent manner (Fig. 7A, B and D). However, the peptide had little effect on GRP78, PERK, eIF- $2 \alpha$, IRE- $1 \alpha$ and caspase-3 protein expression levels, although the peptide at high doses could affect GRP78 and caspase-3 protein expression levels. The results showed that the phosphorylation levels of p-PERK/PERK and p-eIF- $2 \alpha /$ IF- $2 \alpha$ in model group were significantly higher compared with the control group, while PGPIPN reduced the phosphorylation of PERK and eIF- $2 \alpha$ (Fig. 7A and C). Consequently, PGPIPN had the most significant effects on the phosphorylation of PERK/eIF pathway, spliced XBP-1 and cleaved caspase-3 proteins (Fig. 7).

\section{Discussion}

Bioactive peptides can be obtained in natural resources such as milk protein or synthesized by rational design and have been shown to be potential therapeutic agents for a variety 
A

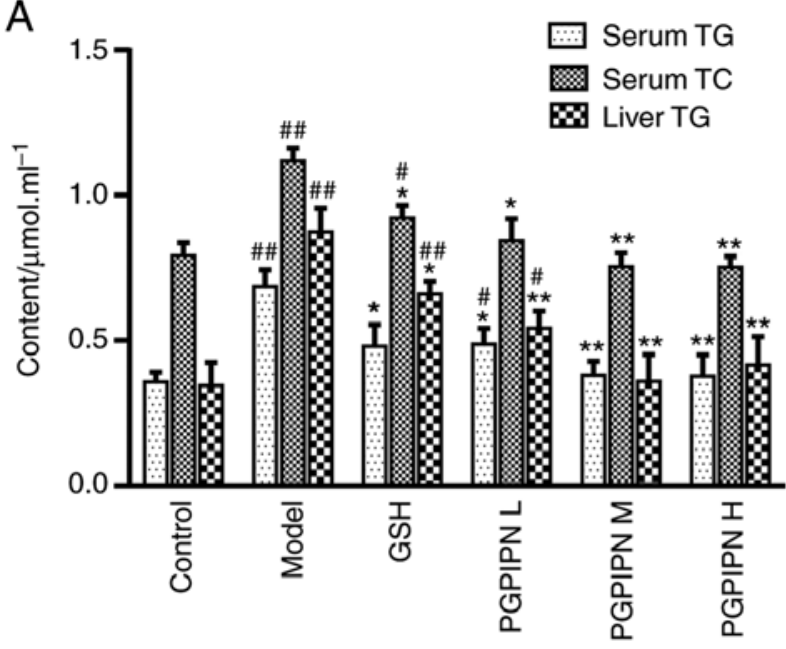

C

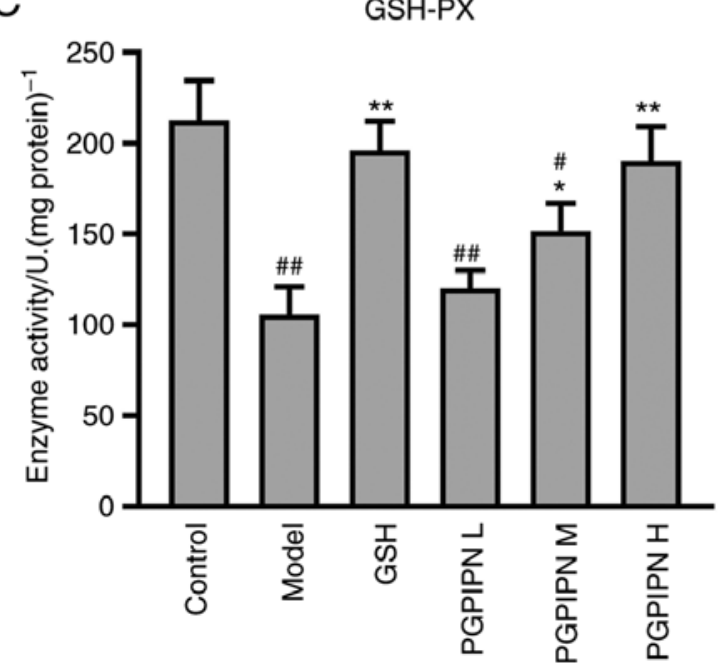

B

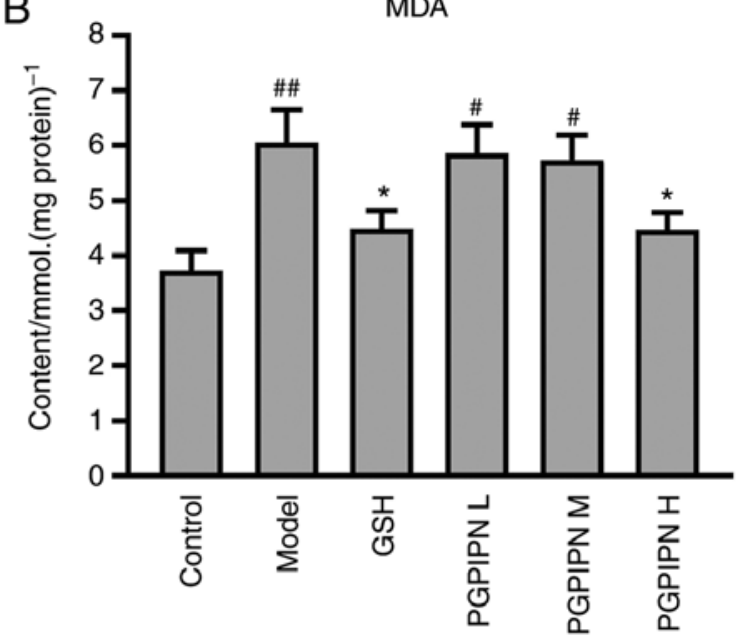

D

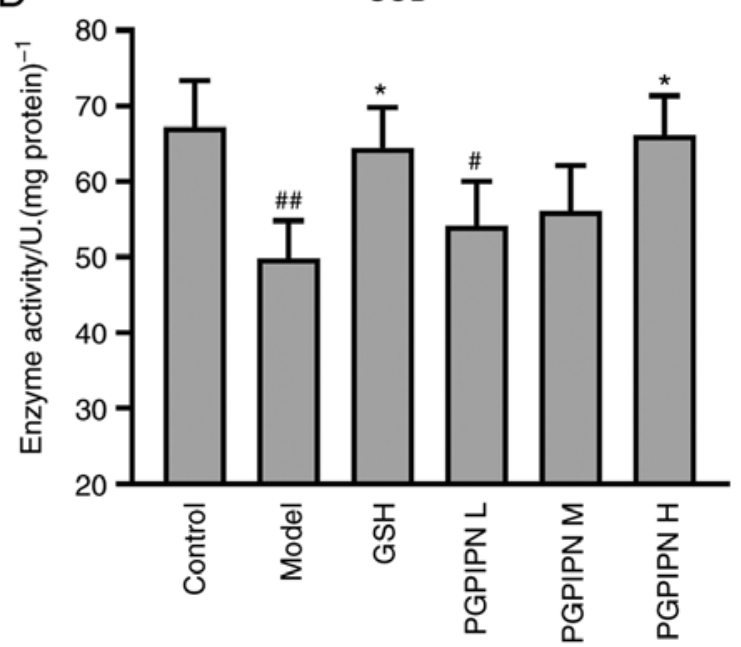

Figure 5. PGPIPN reduces lipid accumulation and attenuated oxidative stress in mice induced with alcohol intake. (A) TG and TC. (B) MDA. (C) GSH-PX. (D) SOD. Data are presented as the mean \pm standard deviation of ten mice in each group. ${ }^{*} \mathrm{P}<0.05$ and ${ }^{* *} \mathrm{P}<0.01$ vs. model. ${ }^{\#} \mathrm{P}<0.05$ and ${ }^{\# \#} \mathrm{P}<0.01$ vs. control. $\mathrm{TG}$, triglyceride; TC, total cholesterol; MDA, malondialdehyde; SOD, superoxide dismutase; GSH-PX, glutathione peroxidase; PGPIPN, Pro-Gly-Pro-Ile-Pro-Asn; $\mathrm{L}$, low dose; $\mathrm{M}$, medium dose; $\mathrm{H}$, high dose.

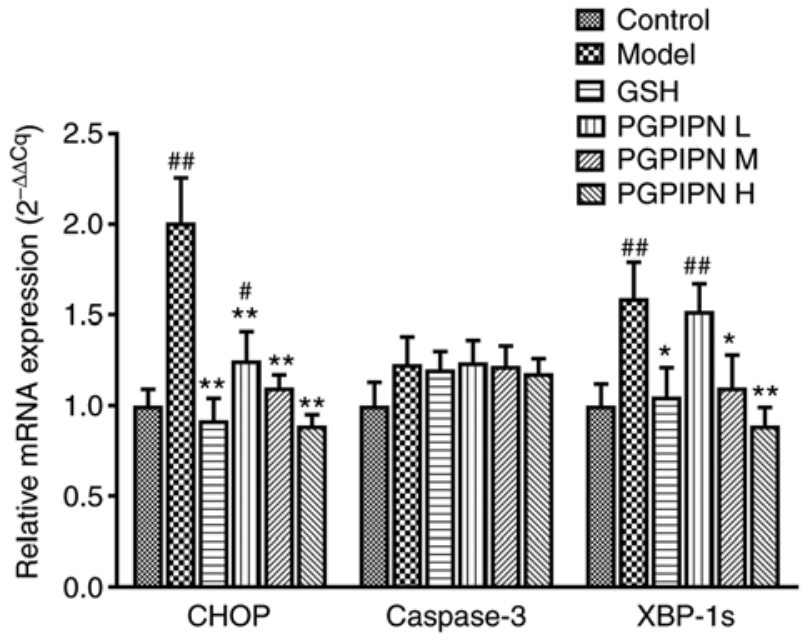

Figure 6. PGPIPN regulates the mRNA expression levels of CHOP, caspase-3 and XBP-1s. $\beta$-actin was used as the reference gene. Data are presented as the mean \pm standard deviation of ten mice in each group. ${ }^{*} \mathrm{P}<0.05$ and ${ }^{* *} \mathrm{P}<0.01$ vs. model. ${ }^{\#} \mathrm{P}<0.05$ and ${ }^{\# \#} \mathrm{P}<0.01$ vs. control. PGPIPN, Pro-Gly-Pro-Ile-Pro-Asn; $\mathrm{CHOP}, \mathrm{C} / \mathrm{EBP}$ homologous protein; XBP-1s, spliced X-box binding protein 1; L, low dose; M, medium dose; H, high dose; GSH, glutathione. of human diseases, including cancer (25). Bioactive peptides have been described as food ingredients that also exert physiological effects, in addition to nutritional value (26). Moreover, peptides are natural amino acids and have less potential toxicity (27). The present results indicated that PGPIPN plays a significant role in alleviating acute alcohol intake-induced liver injury in vivo, suggesting that PGPIPN has a protective effect on AALI.

In the early stages of AALI, TG accumulates in liver cells, leading to the development of fatty liver (28). Oxidative stress and inflammatory response are the main causes of alcoholic liver disease, which increases the severity of fatty liver, oxidizes unsaturated fatty acids, increases the production of lipid peroxidation products and ultimately leads to liver fibrosis by activating stellate cells (29-31). The main cause of AALI is a large intake of alcohol in a short period of time (32). The main metabolism of alcohol ( $90 \%)$ occurs in the liver, and the liver is injured after heavy drinking, which results in an increase in hepatocyte permeability due to cellular oxidative stress or damage (33). ALT and AST are mainly present in the mitochondria of hepatocytes (34). When hepatocytes 


\section{A}
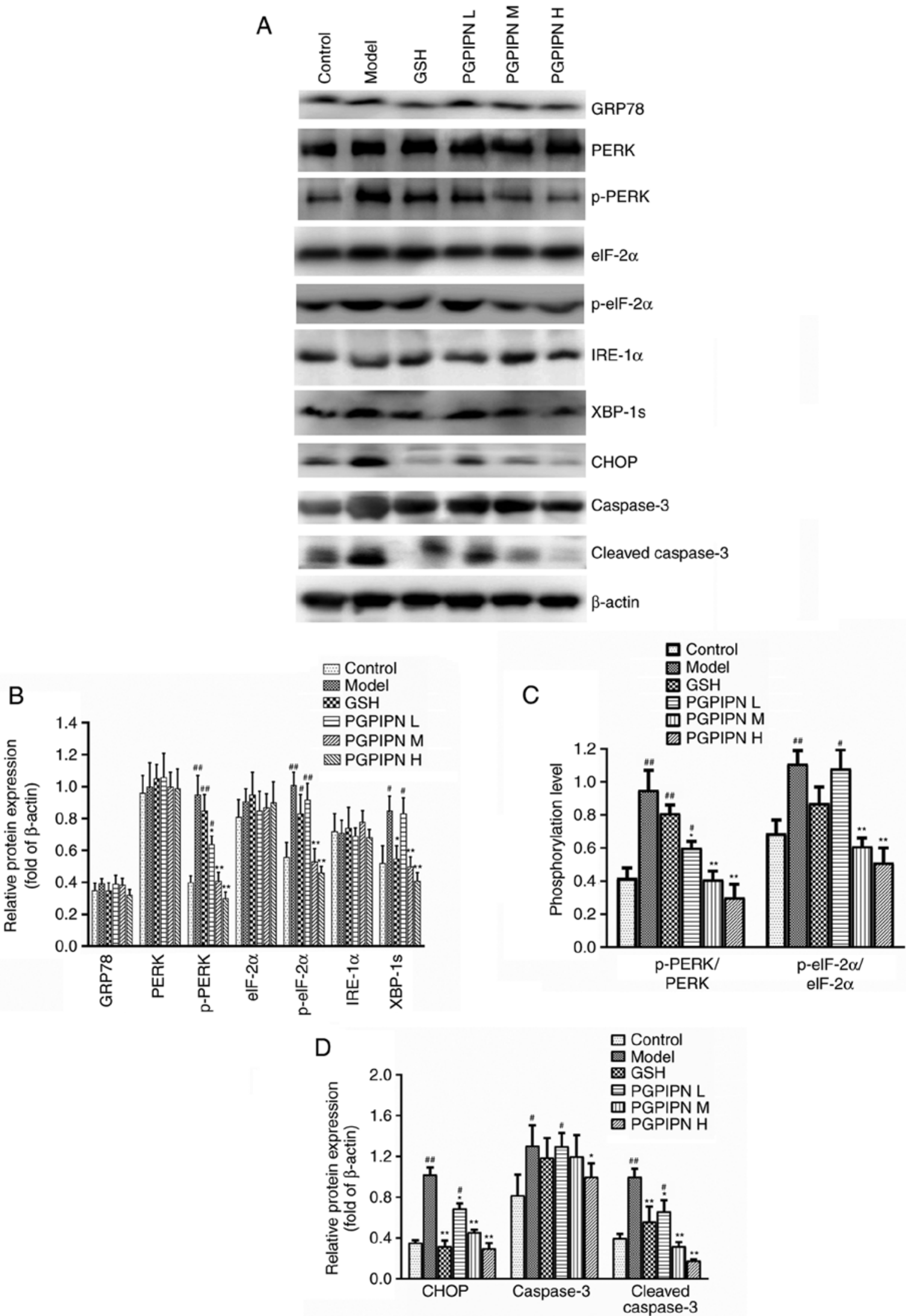

Figure 7. PGPIPN regulates the contents and/or activities of proteins associated with endoplasmic reticulum stress in hepatocytes. (A) GRP78, PERK, p-PERK, eIF-2 $\alpha$, p-eIF-2 $\alpha$, IRE-1 $\alpha$, XBP-1s, CHOP, caspase-3 and cleaved caspase-3 in mice liver tissues in different groups were detected via western blotting. $\beta$-actin was used as the internal reference gene. (B) Relative protein band intensities of GRP78, PERK, p-PERK, eIF-2 $\alpha$, p-eIF-2 $\alpha$, IRE-1 $\alpha$ and XBP-1s. (C) Phosphorylation levels of p-PERK/PERK and p-eIF-2 $\alpha$ /eIF-2 $\alpha$ from relative intensities of protein bands in (A) and normalized to $\beta$-actin. (D) Relative protein band intensities of CHOP, caspase-3 and cleaved caspase-3. $n=10$. Data are presented as the mean \pm standard deviation of ten mice in each group. ${ }^{*} \mathrm{P}<0.05$ and ${ }^{* *} \mathrm{P}<0.01$ vs. model. ${ }^{\#} \mathrm{P}<0.05$ and ${ }^{\# \#} \mathrm{P}<0.01$ vs. control. PGPIPN, Pro-Gly-Pro-Ile-Pro-Asn; CHOP, C/EBP homologous protein; XBP-1s, spliced X-box binding protein 1; p-, phosphorylated; GRP78, 78 kDa glucose-regulated protein; IRE-1 $\alpha$, inositol-requiring enzyme $1 \alpha$; eIF-2 $\alpha$, eukaryotic initiation factor $2 \alpha$; L, low dose; $\mathrm{M}$, medium dose; $\mathrm{H}$, high dose; GSH, glutathione. 
are broken due to AALI, ALT and AST of hepatocytes are rapidly released into the blood, resulting in an increase in serum $(35,36)$. Moreover, alcohol metabolism produces a large amount of acetaldehyde, which induces cell peroxidation to produce MDA (37). The levels of MDA in vivo are an indicator of lipid peroxidation in the body, which can reflect the degree of oxidative stress damage caused by free radical attack (38). The present results demonstrated that PGPIPN effectively reduced MDA levels, enhanced GSH-PX and SOD activities and inhibited alcohol-induced liver oxidative stress damage.

TNF- $\alpha$ is a pro-inflammatory cytokine produced by immune cells (mainly $\mathrm{T}$ lymphocytes), which belongs to a family of both soluble and cell-bound cytokines that has a wide range of biological functions, such as induction of inflammation, apoptosis and lymphatic development (39). IL-1 $\beta$ and IL-6 are two types of cytokines produced by fibroblast, immunocyte and epithelial cells in response to infection and inflammation (40). Furthermore, IL-1 $\beta$ and IL-6 are involved in inflammatory and heat-generating reactions in liver tissues (41). It was reported that decreases in TNF- $\alpha$, IL- $1 \beta$ and IL- 6 levels could reduce inflammation, oxidative stress and apoptosis $(42,43)$. Previous studies showed that pro-inflammatory cytokines such as TNF- $\alpha$, IL-1 $\beta$ and IL-6 play a key role in the development and progression of alcoholic hepatitis $(32,44)$. Moreover, pro-inflammatory cytokines such as TNF- $\alpha$, IL-1 $\beta$ and IL-6 may cause hepatocyte damage $(30,45)$. In the present study, it was found that PGPIPN effectively reduced the expression levels of TNF- $\alpha$, IL- $1 \beta$ and IL- 6 , and then reduced inflammation and hepatocyte damage.

Since ERS often causes unfolded or misfolded proteins to accumulate in the endoplasmic reticulum, the characteristic molecules involved in unfolded protein responses are commonly used to indicate ERS (46). GRP78, as an endoplasmic reticulum resident and chaperone molecule, is a major regulator of ERS response (47). GRP78 regulates the activation of three ER transmembrane binding sensors, IRE1 $\alpha$, cyclic AMP-dependent transcription factor ATF-6 $\alpha$ (ATF6) and PERK, via PERK/eIF-2 $\alpha$ and IRE1 $\alpha$ or ATF6/XBP-1s axes, which are two branches of the unfolded protein response (UPR), affecting the expression of CHOP (48). CHOP was revealed to regulate several pro- and anti-apoptotic genes including Bcl-2, tribbles related protein 3 (TRB3) and growth arrest and DNA damage-inducible protein 34 (GADD34), which are target genes of CHOP (49). The expression of CHOP in normal cells is extremely low; however, $\mathrm{CHOP}$ expression and its accumulation in the nucleus are upregulated during hepatocyte apoptosis induced by ERS (50). Moreover, CHOP does not directly induce apoptosis, but activates caspase-3 to initiate the apoptosis pathway (49). Activated caspase-3 (cleaved caspase-3) specifically cuts proteins associated with cellular activity and triggers the apoptosis cascade reaction, resulting in cell apoptosis (51). The present results suggested that PGPIPN significantly decreased the phosphorylation of the PERK/eIF pathway and levels of spliced XBP-1, resulting in a significant decrease in CHOP. The reduction of $\mathrm{CHOP}$ could decrease the activation of caspase- 3 in liver tissues of mice, thus alleviating ERS. According to the changes in $\mathrm{CHOP}$ and cleaved caspase- 3 levels, PGPIPN could simultaneously downregulate ERS and attenuate acute alcoholic liver cell damage in mice.
ERS regulates proinflammatory cytokine mature and secretion, such as TNF- $\alpha$ and IL-6, the main mechanism of which may be achieved by acting on several signaling pathways, such as the STAT3 pathway, and activating nucleotide-binding oligomerization domain-like receptors $(52,53)$. However, several proinflammatory cytokines can also promote ERS and ERS-related gene expression (54). Our previous studies also showed that PGPIPN exerted immune and antioxidant functions both in vitro and in vivo (data not shown). Fiedorowicz et al (55) reported that some bioactive peptides from bovine caseins could bind the $\mu$-opioid receptor on the cytomembrane to influence the proliferation and cytokine secretion of human peripheral blood mononuclear cells. The changes in the immune response can affect the UPR and some cytokines, especially the expression levels of UPR target genes such as IRE1 $\alpha$ and ATF6. Therefore, it was speculated that PGPIPN may regulate cell signal transduction and alter the expression level or activity of proteins associated with ERS in hepatocytes; however, this requires further investigation. Moreover, the present results suggested that PGPIPN may be a novel safe therapeutic agent for the treatment or prevention of AALI and its related complications.

\section{Acknowledgements}

The authors thank Mr Guanjun Chen of the Center for Scientific Research of Anhui Medical University for valuable help in our experiment. The authors would like to thank Mr Hao Li of the First Affiliated Hospital of Anhui Medical University for help in the assessment, sampling and pathological examination of alcoholic liver injury from liver tissues of model mice.

\section{Funding}

The study and the preparation of the paper were funded by the National Natural Science Foundation of China (grant nos. 81472448 and 81601107 ) and the National College Students Innovation and Entrepreneurship Training Program of China (grant no. 201910366004).

\section{Availability of data and materials}

The datasets used and/or analyzed during the current study are available from the corresponding author on reasonable request.

\section{Authors' contributions}

YQ conceived the idea, designed the study, contributed reagents and materials and wrote the manuscript. QX, HX and $\mathrm{XC}$ performed research and analyzed data. PW, JL, WW and FG performed research. YX performed statistical analyses and revised the manuscript. All authors read and approved the final manuscript.

\section{Ethics approval and consent to participate}

All animal experiments were performed under procedures approved by the Institutional Animal Care and Use Committee of Anhui Medical University (approval no. LLSC20180132). 


\section{Patient consent for publication}

Not applicable.

\section{Competing interests}

The authors declare that they have no competing interests.

\section{References}

1. Marroni CA, Fleck AM Jr, Fernandes SA, Galant LH, Mucenic M de Mattos Meine MH, Mariante-Neto G and Brandão ABM: Liver transplantation and alcoholic liver disease: History, controversies, and considerations. World J Gastroenterol 24 2785-2805, 2018.

2. Jiang Z, Wang J, Xue H, Wang M, Jiang H, Liang Y, Dias AC, Gregory M, Chen C and Zhang X: Protective effect of wild Corni fructus methanolic extract against acute alcoholic liver injury in mice. Redox Rep 22: 338-345, 2017.

3. Louvet A and Mathurin P: Alcoholic liver disease: Mechanisms of injury and targeted treatment. Nat Rev Gastroenterol Hepatol 12: 231-242, 2015.

4. O'Shea RS, Dasarathy S and McCullough AJ; Practice Guideline Committee of the American Association for the Study of Liver Diseases; Practice Parameters Committee of the American College of Gastroenterology: Alcoholic liver disease. Hepatology 51: 307-328, 2010.

5. Torruellas C, French SW and Medici V: Diagnosis of alcoholic liver disease. World J Gastroentero 20: 11684-11699, 2014.

6. Stickel F, Datz C, Hampe J and Bataller R: Erratum: Pathophysiology and management of alcoholic liver disease: Update 2016. Gut Liver 11: 447, 2017.

7. Liu Y, Chen X, Qiu M, Chen W, Zeng Z and Chen Y: Emodin ameliorates ethanol-induced fatty liver injury in mice. Pharmacology 94: 71-77, 2014.

8. Stickel F, Datz C, Hampe J and Bataller R: Pathophysiology and management of alcoholic liver disease: Update 2016. Gut Liver 11: 173-188, 2017.

9. Marcone S, Haughton K, Simpson PJ, Belton O and Fitzgerald DJ: Milk-derived bioactive peptides inhibit human endothelial-monocyte interactions via PPAR- $\gamma$ dependent regulation of NF- $\mathrm{B}$. J Inflamm (Lond) 12: 1, 2015.

10. Saadi S, Saari N, Anwar F, Abdul Hamid A and Ghazali HM: Recent advances in food biopeptides: Production, biological functionalities and therapeutic applications. Biotechnology Advances 33: 80-116, 2015.

11. Gokhale AS and Satyanarayanajois S: Peptides and peptidomimetics as immunomodulators. Immunotherapy 6: 755-774, 2014.

12. Lonnerdal B: Nutritional and physiologic significance of human milk proteins. Am J Clin Nutr 77: 1537S-1543S, 2003.

13. Qi N, Liu C, Yang H, Shi W, Wang S, Zhou Y, Wei C, Gu F and Qin Y: Therapeutic hexapeptide (PGPIPN) prevents and cures alcoholic fatty liver disease by affecting the expressions of genes related with lipid metabolism and oxidative stress. Oncotarget 8 : 88079-88093, 2017.

14. Wu Y, Pan X, Zhang S, Wang W, Cai M, Li Y, Yang F and Guo H: Protective effect of corn peptides against alcoholic liver injury in men with chronic alcohol consumption: A randomized double-blind placebo-controlled study. Lipids Health Dis 13: $192,2014$.

15. Meisel H: Biochemical properties of regulatory peptides derived from milk proteins. Biopolymers 43: 119-128, 1997.

16. Gevaert B, Veryser L, Verbeke F, Wynendaele E and De Spiegeleer B: Fish hydrolysates: A regulatory perspective of bioactive peptides. Protein Pept Lett 23: 1052-1060, 2016.

17. Kanwar JR, Kanwar RK, Sun X, Punj V, Matta H, Morley SM, Parratt A, Puri M and Sehgal R: Molecular and biotechnological advances in milk proteins in relation to human health. Curr Protein Pept Sci 10: 308-338, 2009.

18. GillHS, Doull F, Rutherfurd KJ and Cross ML: Immunoregulatory peptides in bovine milk. Br J Nutr 84 (Suppl 1): S111-S117, 2000

19. Fiat AM, Migliore-Samour D, Jolles P, Drouet L, Bal dit Sollier C and Caen J: Biologically active peptides from milk proteins with emphasis on two examples concerning antithrombotic and immunomodulating activities. J Dairy Sci 76: 301-310, 1993.
20. Meisel $\mathrm{H}$ and FitzGerald RJ: Biofunctional peptides from milk proteins: Mineral binding and cytomodulatory effects. Curr Pharm Des 9: 1289-1295, 2003.

21. Zhao M, Wei C, Yang X, Zhou J, Wang J, Gu F, Lei T and Qin Y: The milk-derived hexapeptide PGPIPN inhibits the invasion and migration of human ovarian cancer cells by regulating the expression of MTA1 and NM23H1 genes. Int J Oncol 48: 1721-1729, 2016.

22. Livak KJ and Schmittgen TD: Analysis of relative gene expression data using real-time quantitative PCR and the 2(-Delta Delta C(T)) method. Methods 25: 402-408, 2001.

23. Green MR, Sambrook J and Sambrook J: Molecular cloning: A laboratory manual. Cold Spring Harbor Laboratory Press, Cold Spring Harbor, NY, 2012.

24. Lv SX and Qiao X: Isovitexin (IV) induces apoptosis and autophagy in liver cancer cells through endoplasmic reticulum stress. Biochem Biophys Res Commun 496: 1047-1054, 2018.

25. Wang W, Gu F, Wei C, Tang Y, Zheng X, Ren M and Qin Y: PGPIPN, a therapeutic hexapeptide, suppressed human ovarian cancer growth by targeting BCL2. PLoS One 8: e60701, 2013.

26. Phelan M and Kerins D: The potential role of milk-derived peptides in cardiovascular disease. Food Funct 2: 153-167, 2011.

27. Kreider RB, Iosia M, Cooke M, Hudson G, Rasmussen C, Chen $\mathrm{H}$, Mollstedt $\mathrm{O}$ and Tsai $\mathrm{MH}$ : Bioactive properties and clinical safety of a novel milk protein peptide. Nutr J 10: 99, 2011.

28. Purohit V, Gao B and Song BJ: Molecular mechanisms of alcoholic fatty liver. Alcohol Clin Exp Res 33: 191-205, 2009.

29. You M, Jogasuria A, Taylor C and Wu J: Sirtuin 1 signaling and alcoholic fatty liver disease. Hepatobiliary Surg Nutr 4: 88-100, 2015.

30. Qu BG, Wang H, Jia YG, Su JL, Wang ZD, Wang YF, Han XH, Liu YX, Pan JD and Ren GY: Changes in tumor necrosis factor-alpha, heat shock protein 70 , malondialdehyde, and superoxide dismutase in patients with different severities of alcoholic fatty liver disease: A prospective observational study. Medicine Baltimore) 94: e643, 2015.

31. Abdelmegeed MA, Banerjee A, Jang S, Yoo SH, Yun JW, Gonzalez FJ, Keshavarzian A and Song BJ: CYP2E1 potentiates binge alcohol-induced gut leakiness, steatohepatitis, and apoptosis. Free Radic Biol Med 65: 1238-1245, 2013.

32. Zhou JY, Jiang ZA, Zhao CY, Zhen Z, Wang W and Nanji AA: Long-term binge and escalating ethanol exposure causes necroinflammation and fibrosis in rat liver. Alcohol Clin Exp Res 37: 213-222, 2013.

33. Cederbaum AI: Alcohol metabolism. Clin Liver Dis 16: 667-685, 2012.

34. Ding $\mathrm{H}$ and Wen $\mathrm{Z}$ : Overexpression of $\mathrm{C}$-sis inhibits $\mathrm{H} 2 \mathrm{O} 2$-induced Buffalo rat liver cell apoptosis in vitro and alleviates liver injury in a rat model of fulminant hepatic failure. Int J Mol Med 42: 873-882, 2018.

35. Xing H, Jia K, He J, Shi C, Fang M, Song L, Zhang P, Zhao Y, Fu J and Li S: Establishment of the tree shrew as an alcohol-induced fatty liver model for the study of alcoholic liver diseases. PLoS One 10: $\mathrm{e} 0128253,2015$.

36. He Y, Liu Q, Li Y, Yang X, Wang W, Li T, Zhang W, Cui Y, Wang $C$ and Lin R: Protective effects of hydroxysafflor yellow A (HSYA) on alcohol-induced liver injury in rats. J Physiol Biochem 71: 69-78, 2015.

37. Kimura M, Yokoyama A and Higuchi S: Aldehyde dehydrogenase-2 as a therapeutic target. Expert Opin Ther Targets 23: 955-966, 2019.

38. Ding RB, Tian K, Cao YW, Bao JL, Wang M, He C, Hu Y, Su H and Wan JB: Protective effect of panax notoginseng saponins on acute ethanol-induced liver injury is associated with ameliorating hepatic lipid accumulation and reducing ethanol-mediated oxidative stress. J Agric Food Chem 63: 2413-2422, 2015.

39. Mehaffey E and Majid DSA: Tumor necrosis factor- $\alpha$, kidney function, and hypertension. Am J Physiol Renal Physiol 313: F1005-F1008, 2017.

40. Slaats J, Ten Oever J, van de Veerdonk FL and Netea MG: IL-1 $\beta /$ IL-6/CRP and IL-18/ferritin: Distinct inflammatory programs in infections. PLoS Pathog 12: e1005973, 2016.

41. Unver N and McAllister F: IL-6 family cytokines: Key inflammatory mediators as biomarkers and potential therapeutic targets. Cytokine Growth Factor Rev 41: 10-17, 2018.

42. Wang H, Li J, Gai Z, Kullak-Ublick GA and Liu Z: TNF- $\alpha$ deficiency prevents renal inflammation and oxidative stress in obese mice. Kidney Blood Press Res 42: 416-427, 2017. 
43. Yang L, Sun YY, Liu YR, Yin NN, Bu FT, Yu HX, Du XS $\mathrm{Li} \mathrm{J}$ and Huang C: PTP1B promotes macrophage activation by regulating the NF-KB pathway in alcoholic liver injury. Toxicol Lett 319: 11-21, 2020.

44. Lee JS, An Y, Yoon CJ, Kim JY, Kim KH, Freeman AF, Yim JJ, Shin EC, Holland SM, Lee EY and Ju YS: Germline gain-of-function mutation of STAT1 rescued by somatic mosaicism in immune dysregulation-polyendocrinopathy-enteropathy-X-linked-like disorder. J Allergy Clin Immunol 145: $1017-1021,2020$

45. Starkel P, Schnabl B, Leclercq S, Komuta M, Bataller R, Argemi J, Palma E, Chokshi S, Hellerbrand C, Maccioni L, et al Deficient IL-6/Stat 3 signaling, high TLR7, and type I interferons in early human alcoholic liver disease: A triad for liver damage and fibrosis. Hepatol Commun 3: 867-882, 2019.

46. Senft D and Ronai ZA: UPR, autophagy, and mitochondria crosstalk underlies the ER stress response. Trends Biochem Sci 40: 141-148, 2015.

47. Shimizu A, Kaira K, Yasuda M, Asao T and Ishikawa O: Clinical and pathological significance of er stress marker (BiP/GRP78 and PERK) expression in malignant melanoma. Pathol Oncol Res 23: 111-116, 2017.

48. Kouznetsova VL, Hu H, Teigen K, Zanetti M and Tsigelny IF: Cripto stabilizes GRP78 on the cell membrane. Protein Sci 27: 653-661, 2018.

49. Li Y, Guo Y, Tang J, Jiang J and Chen Z: New insights into the roles of CHOP-induced apoptosis in ER stress. Acta Biochim Biophys Sin (Shanghai) 46: 629-640, 2014.
50. Malhi $\mathrm{H}$ and Kaufman RJ: Endoplasmic reticulum stress in liver disease. J Hepatol 54: 795-809, 2011

51. Jo HJ, Yang JW, Park JH, Choi ES, Lim CS, Lee S and Han CY: Endoplasmic reticulum stress increases DUSP5 expression via PERK-CHOP pathway, leading to hepatocyte death. Int J Mol Sci 20: 4369, 2019.

52. Yakin M, Seo B and Rich A: Tunicamycin-induced endoplasmic reticulum stress up-regulates tumour-promoting cytokines in oral squamous cell carcinoma. Cytokine 120: 130-143, 2019.

53. Chen HP, Zhou Y, Qin XF, Wang L, Lin XF, Chen H and Hu YB: Endoplasmic reticulum stress regulates autophagy and tumor necrosis factor- $\alpha$ secretion of RAW264.7 cells induced by silica. Zhonghua Lao Dong Wei Sheng Zhi Ye Bing Za Zhi 38: 91-95, 2020 (In Chinese).

54. Pinto AP, da Rocha AL, Cabrera EMB, Marafon BB, Kohama EB, Rovina RL, Simabuco FM, Bueno Junior CR, de Moura LP, Pauli JR, et al: Role of interleukin-6 in inhibiting hepatic autophagy markers in exercised mice. Cytokine 130: 155085, 2020.

55. Fiedorowicz E, Jarmolowska B, Iwan M,Kostyra E, Obuchowicz R and Obuchowicz M: The influence of $\mu$-opioid receptor agonist and antagonist peptides on peripheral blood mononuclear cells (PBMCs). Peptides 32: 707-712, 2011.

(i) $\Theta$ This work is licensed under a Creative Commons Attribution-NonCommercial-NoDerivatives 4.0 International (CC BY-NC-ND 4.0) License. 